\title{
A new approach to disease-modifying drug trials in Parkinson's disease
}

\author{
Roger A. Barker, ${ }^{1}$ Mark Stacy, ${ }^{2}$ and Patrik Brundin ${ }^{3,4}$
}

\begin{abstract}
1Department of Neurology and Clinical Neurosciences, Cambridge Center for Brain Repair, University of Cambridge, Cambridge, United Kingdom. ${ }^{2}$ Duke University School of Medicine, Durham, North Carolina, USA. ${ }^{3}$ Center for Neurodegenerative Science, Van Andel Institute, Grand Rapids, Michigan, USA. ${ }^{4}$ Neuronal Survival Unit, Department of Experimental Medical Science, Wallenberg Neuroscience Center, Lund University, Lund, Sweden.
\end{abstract}

\begin{abstract}
Translating new findings in the laboratory into therapies for patients is a slow and expensive process. The development of therapies for neurodegenerative diseases is further complicated by the difficulty in determining whether the drug truly retards the slow degenerative process or provides only symptomatic benefit. In this issue, Aviles-Olmos et al. describe a first in Parkinson's disease (PD) patient study using a drug previously approved for diabetes treatment. In addition to suggesting that the drug may indeed be disease modifying in PD, their innovative approach suggests there may be more rapid and inexpensive avenues for testing novel therapies in PD.
\end{abstract}

Taking new potentially disease-modifying therapies from studies in experimental animals to the clinic in patients with chronic neurodegenerative disorders requires a balance between optimal trial design and expense, and new methods are desperately needed. In this issue, Aviles-Olmos and colleagues describe a novel approach toward exploring the potential for diseasemodifying effects in patients with Parkinson's disease (PD) using a drug approved for the treatment of type 2 diabetes (1). They examined the drug exenatide, a glucagon-like peptide-1 (GLP-1) receptor agonist that has demonstrated promise in several animal models of PD (2). This work is remarkable not only because it is an attempt to examine the effectiveness of repurposing a drug that is already in clinical use, but also because it represents a cost-effective method. This is important because the clinical research community faces a challenge in developing proof of concept studies in a way that gives confidence to proceed to the necessary - and markedly more costly - double-blind, placebo-controlled trials.

Conflict of interest: Roger A. Barker sits on the Research Advisory Board of the Cure Parkinson's Trust. Patrik Brundin collaborates with Metabolic Solutions Development Company LLC on a project funded by the Michael J. Fox Foundation. Mark Stacy receives research support from Ceregene, Parkinson Study Group, IMPAX, and the Michael J. Fox Foundation and is a consultant for Allergan, Chelsea, Merz, Osmotica Biotie, General Electric, GlaxoSmithKline, Merck, Neuronova, Novartis, Noven, SK Life Sciences, TEVA, and UCB.

Citation for this article: J Clin Invest. 2013; 123(6):2364-2365. doi:10.1172/JCI69690.

\section{Targeting the dopaminergic pathway in PD}

PD affects about 1 in 800 people, with an average age of onset of around 65-70 years (3). The disease typically presents with a resting tremor, bradykinesia, and rigidity. The core pathology underlying these motor symptoms and signs is the progressive degeneration of the nigrostriatal dopaminergic pathway. However, it is now well recognized that the disease is much more than this, and patients are frequently also troubled by an array of nonmotor symptoms (e.g., depression, cognitive decline, constipation, and pain), and pathological intraneuronal protein aggregates called Lewy bodies and Lewy neurites also develop in a wide range of brain areas as the disease progresses (4).

Since targeting the dopaminergic pathway pharmacologically is of significant benefit to patients, there has been great interest in trying to restore this system in the form of dopamine cell replacement (5), delivery of neurotrophic factors $(6,7)$, or neuroprotective drug treatment $(7,8)$. Because exenatide has been shown to reduce neurodegeneration in several different neurotoxic lesion rodent models of PD (such as those induced by MPTP, lipopolysaccharide, $\mathrm{N}$-[2-chloroethyl]-N-ethyl-2-bromobenzylamine [DSP-4], parachloroampetamine [pCA], and 6-hydroxydopamine), this agent has promise in an area of great unmet need (9-11). As with other studies of neuroprotective agents in animal models of $\mathrm{PD}$, exenatide has been administered in close association with the toxin, and the treat- ment has been found to reduce neuronal loss. Exactly how it mediates this effect is still unresolved, but it might involve direct protective effects on different monoaminergic neurons (2, 9-11), increased cell proliferation in the adult brain (12), or indirect actions on inflammation $(2,9)$.

In a clinical setting, it is difficult to assess whether an agent has truly rescued dopamine neurons or simply provided symptomatic benefit. In clinical trials with neurotrophic factors and other potentially neuroprotective treatments, this problem has been tackled by imaging the nigrostriatal dopamine pathway and using long follow-up periods, both of which bring substantial cost. In the case of drug therapies, the use of delayed-start trials has been advocated. In this approach, there is a delay in the introduction of the active agent in one arm of the study, with the aim of determining whether subjects on the treatment for a long period reach the same end point as those on it for a shorter time. If the drug provides solely symptomatic benefit, all patients would be expected to reach the same end point regardless of when the drug was started. As an alternative to this approach, patients may be reassessed after a defined washout period for the active agent at the end of the trial, with the aim of washing away any symptomatic effects of the drug to see whether there are any true underlying, neuroprotective effects (13-15).

\section{Trial design}

Repurposing a drug for treating a novel disease is difficult, particularly with an injectable therapy. While hospital pharmacies are capable of masking approved tablets to look the same as a placebo tablet, this is not possible with an injectable device. So what does one do? How can one start to take such therapies to clinic with the hope of seeing an effect that merits further investigation in the absence of a large, expensive, properly powered double-blind, placebo-controlled trial? Here, the investigators studied a par- 
allel group of patients that were matched to those in the treatment arm, but did not receive any injectable therapy (1). This approach obviously lacks the rigor of a placebo-controlled trial, but does allow one to draw conclusions about how the intervention fares against best medical therapy in patients with PD at a similar stage of illness.

The standard approach with any therapy is to do a first-into-human phase I study and then move to phase II trials to ascertain whether the agent is well tolerated and exhibits some efficacy. An approved drug may enter the regulatory pathway later in the process. In this trial, Aviles-Olmos et al. demonstrated that exenatide is well tolerated in PD (1); given the large body of safety data already gathered from its use in the diabetes population, exenatide may now perhaps be ready for the phase III, pivotal trial stage of testing. Phase II testing also assesses efficacy, and in the present study, the investigators were challenged to explore whether exenatide might modify the slowly evolving progressive course of $\mathrm{PD}$, rather than simply have some direct symptomatic effect (1). In an effort to reduce investigator bias, Aviles-Olmos et al. used an established procedure in which participants are videotaped and assessed by a blinded third party (16).

In the absence of a placebo group or any reliable peripheral measure that tracks the pathology of $\mathrm{PD}$, how could the investigators at least get some indication of whether exenatide might have a disease-modifying effect? Imaging the dopaminergic pathway using F-dopa PET is extremely expensive (approximately $\$ 9,000$ per scan), but reliably measures signals over a short period of time. However, functional imaging is not always straightforward, and interpreting changes in F-dopa PET can be difficult $(15,17,18)$. Using ${ }^{123}$ I-FP-CIT SPECT scanning may offer a cheaper, more feasible approach, but it lacks the sensitivity to detect gradual changes in the network once the disease process has begun (19).

Aviles-Olmos and coworkers chose to treat participants with exenatide for twelve months, and then reassess them after a washout of two months, with the assumption that any symptomatic effects would have been lost by the time the patient was reevaluated. The study team saw a sustained benefit two months after the exenatide was discontinued, including in a number of motor measures as well as nonmotor measures. Notably, cognitive scores were improved by exenatide (1). Although a sustained symptomatic effect potentially could explain the improvement after exenatide was discontinued, the observations suggest that exenatide may truly have slowed disease progression. However, without a placebo injectable study arm, one cannot entirely exclude that there was a protracted placebo effect after cessation of the drug therapy. Indeed, protracted placebo effects have been seen in some gene therapy trials while patients have still been blinded $(20,21)$. In the present study, one would predict that if a placebo effect played a major role, it ought to have waned during the final two months, when the patients were aware that they were no longer receiving injections of the active drug.

\section{Conclusions and future directions}

What are the conclusions that one can draw from this study in the absence of a placebo arm? The study clearly shows, using a number of well-established standard measures, that the group receiving exenatide improved over fourteen months and that the patients not receiving the drug declined. This could be due to an acute treatment effect or, as discussed above, a placebo effect (16). However, the very mild symptomatic decline during the washout period speaks more in favor of a disease-modifying effect. In short, this study demonstrated that a drug in clinical use for another medical condition can successfully be tested for its disease-modifying effects in patients with PD using standard clinical measures, without biomarkers and without a placebo-controlled arm, in a relatively inexpensive way. Obviously, any results can only be seen as providing a signal of possible efficacy that is worth pursuing further. As with any experiment, replication is the first step in proving that a result is true, and a double-blind, placebo-controlled trial will ultimately be required.

Perhaps the most important feature of this study is that it has adopted a pragmatic approach to the problems of taking established drugs to new areas of therapy in a neurodegenerative disorder and done so with a study design that is informative and affordable. This is critical in an environment in which costs for drug trials have escalated and have become a significant hurdle. The authors are to be congratulated for their efforts, although establishing whether this agent truly can modify the progression in PD will require further work.

Address correspondence to: Patrik Brundin, Center for Neurodegenerative Science, Van Andel Institute, 333 Bostwick
Avenue N.E., Grand Rapids, Michigan 49503, USA. Phone: 616.234.5684; E-mail: patrik.brundin@vai.org.

1. Aviles-Olmos I, et al. Exenatide and the treatment of patients with Parkinson's disease. J Clin Invest. 2013;123(6):2730-2736.

2. Harkavyi A, Whitton PS. Glucagon-like peptide 1 receptor stimulation as a means of neuroprotection. Br J Pharmacol. 2010;159(3):495-501.

3. Schapira AH, Jenner P. Etiology and pathogenesis of Parkinson's disease. Mov Disord. 2011; 26(6):1049-1055

4. Dickson DW, et al. Neuropathological assessment of Parkinson's disease: refining the diagnostic criteria. Lancet Neurol. 2009;8(12):1150-1157.

5. Barker RA, Barrett J, Mason SL, Bjorklund A. Fetal dopaminergic transplantation trials and the future of neural grafting in Parkinson's disease. Lancet Neurol. 2013;12(1):84-91.

6. Kordower JH, Bjorklund A. Trophic factor gene therapy for Parkinson's disease. Mov Disord. 2013; 28(1):96-109

7. Bjorklund T, Kordower JH. Gene therapy for Parkinson's disease. Mov Disord. 2010; 25(suppl 1):S161-S173.

8. de la Fuente-Fernández R, Schulzer M, Mak E, Sossi $\mathrm{V}$. Trials of neuroprotective therapies for Parkinson's disease: problems and limitations. Parkinsonism Relat Disord. 2010;16(6):365-369.

9. Harkavyi A, Abuirmeileh A, Lever R, Kingsbury AE, Biggs CS, Whitton PS. Glucagon-like peptide 1 receptor stimulation reverses key deficits in distinct rodent models of Parkinson's disease. J Neuroinflammation. 2008;5:19.

10. Li Y, et al. GLP-1 receptor stimulation preserves primary cortical and dopaminergic neurons in cellular and rodent models of stroke and Parkinsonism. Proc Natl Acad Sci U S A. 2009;106(4):1285-1290.

11. Rampersaud N, Harkavyi A, Giordano G, Lever R, Whitton J, Whitton PS. Exendin-4 reverts behavioural and neurochemical dysfunction in a pre-motor rodent model of Parkinson's disease with noradrenergic deficit. Br J Pharmacol. 2012; 167(7):1467-1479.

12. Bertilsson G, et al. Peptide hormone exendin-4 stimulates subventricular zone neurogenesis in the adult rodent brain and induces recovery in an animal model of Parkinson's disease. J Neurosci Res. 2008;86(2):326-338.

13. Olanow CW, et al. A randomized, double-blind, placebo-controlled, delayed start study to assess rasagiline as a disease modifying therapy in Parkinson's disease (the ADAGIO study): rationale, design, and baseline characteristics. Mov Disord. 2008;23(15):2194-2201.

14. Olanow CW, et al. A double-blind, delayed-start trial of rasagiline in Parkinson's disease. $N$ Engl J Med. 2009;361(13):1268-1278.

15. Fahn S, et al. Levodopa and the progression of Parkinson's disease. NEngl J Med. 2004;351(24):2498-2508.

16. Galpern WR, et al. Sham neurosurgical procedures in clinical trials for neurodegenerative diseases: scientific and ethical considerations. Lancet Neurol. 2012;11(7):643-650.

17. Gill SS, et al. Direct brain infusion of glial cell line-derived neurotrophic factor in Parkinson disease. Nat Med. 2003;9(5):589-595.

18. Whone AL, et al. Slower progression of Parkinson's disease with ropinirole versus levodopa: The REALPET study. Ann Neurol. 2003;54(1):93-101.

19. Breen DP, Rowe JB, Barker RA. Role of brain imaging in early parkinsonism. BMJ. 2011;342:d638.

20. LeWitt PA, et al. AAV2-GAD gene therapy for advanced Parkinson's disease: a double-blind, shamsurgery controlled, randomised trial. Lancet Neurol. 2011;10(4):309-319.

21. Marks WJ Jr, et al. Gene delivery of AAV2-neurturin for Parkinson's disease: a double-blind, randomised, controlled trial. Lancet Neurol. 2010;9(12):1164-1172. 